CLAUDIA PATRICIA SERNA-GIRALDO

\title{
AVALIAÇÃO DE JUNTAS SOLDADAS DE AÇOS CARBONO ATRAVÉS DO RUÍDO MAGNÉTICO DE BARKHAUSEN
}

Tese apresentada à Escola Politécnica da Universidade de São Paulo como requisito para a obtenção do Título de Doutora em Engenharia

SÃo PAULO

2007 
CLAUDIA PATRICIA SERNA-GIRALDO

\section{AVALIAÇÃO DE JUNTAS SOLDADAS DE AÇOS CARBONO ATRAVÉS DO RUÍDO MAGNÉTICO DE BARKHAUSEN}

Tese apresentada à Escola Politécnica da Universidade de São Paulo como requisito para a obtenção do Título de Doutora em Engenharia

Área de concentração:

Engenharia Mecânica

Orientador:

Professor Doutor Linilson Rodrigues Padovese

SÃO PAULO

2007 


\section{FICHA CATALOGRÁFICA}

\section{Serna-Giraldo, Claudia Patricia}

Avaliação de juntas soldadas de aços carbono através do ruído magnético de Barkhausen / C.P. Serna-Giraldo. -- São Paulo, 2007.

$140 \mathrm{p}$.

Tese (Doutorado) - Escola Politécnica da Universidade de São Paulo. Departamento de Engenharia Mecânica.

1.Ruído magnético de Barkhausen 2.Ensaios não destrutivos magnéticos 3. Magnetização em ferromagnéticos 4.Soldagem de aços carbono I.Universidade de São Paulo. Escola Politécnica. Departamento de Engenharia Mecânica II.t. 


\section{DEDICATÓRIA}

Despertar cada amanecer Con uma linda sonrisa llena de amor

Ilumina todos mis días. A mis dos grandes amores:

Nelson y Santiago 


\section{AGRADECIMENTOS}

Para finalizar pequenas e grandes obras é sempre necessário a ajuda de mãos amigas e mentes abertas e experientes. Muitas pessoas me acompanharam neste percurso e hoje sou muito grata a elas.

- Ao meu orientador, professor Dr. Linilson Rodrigues Padovese, sua sensibilidade e confiança se tornaram a chave para a conclusão do meu trabalho.

- A CAPES pelo apoio através da bolsa de Doutorado.

- Aos meus colegas do Departamento de Ingeniería Metalúrgica y de Materiales e aos diretivos da Facultad de Ingeniería de la Universidad de Antioquia de Colombia, pelo apoio que me brindaram com a concessão da comissão de estudos para que eu pudesse terminar o meu doutorado.

- Aos professores da Universidad de Oriente de Cuba: Dr. José Anglada, Ms. José Pérez Benitez, Ms. Manuel Alberteris e muito especialmente ao professor Dr. Julio Capó que esteve sempre presente me ajudando a entender, me ensinando e discutindo sobre o magnetismo e o ruído magnético de Barkhausen.

- Aos funcionários do Departamento de Engenharia Mecânica da Escola Politécnica da USP pelo apoio logístico.

- Aos meus colegas do LADIN: Carlos, Elvys, Freddy, Márcia, Ricardo, Rodrigo, Rui e Wilson Carlos pelo companheirismo.

- Ao Departamento de Engenharia Metalúrgica de Materiais da EPUSP, e seus técnicos Cláudio, Danilo, Lívio e Rubens pela disponibilidade dos equipamentos da oficina mecânica, da metalografia e dos tratamentos térmicos no início deste projeto.

- Ao LFS e seus técnicos Francisco, Jovanio e Leandro pela disponibilidade do laboratório de preparação metalográfica, do microdurômetro, do microscópio óptico e da lupa.

- Ao Laboratório de Soldagem da Universidad Nacional de Colombia, em especial ao professor MSc. Jorge Giraldo e ao técnico Jorge Atehortúa pela elaboração dos corpos de prova soldados.

- Ao Laboratório de Fundição e de Tratamentos Térmicos da Universidad de Antioquia de Colombia, pela análise de composição química e os tratamentos térmicos das amostras. 
- Ao Dr. Paulo Massoti do IPEN pelas sugestões para as análises com a transformada wavelet.

- Ao professor Dr. Rodrigo Magnabosco da FEI pelos tratamentos térmicos a vácuo.

- Aos professores Dr. Nelson de Lima e Dr. Luis Gallego do IPEN pela difração de raios-X.

- Ao Dr. Marcelo Lancarotte pelas discussões e apoio no início do projeto.

- Ao Nelson e o Santiago, que estiveram sempre do meu lado e no meu coração me fortalecendo, me incentivando com seu amor, para juntos ver meu sonho realizado.

- A minha família pelo amor e incentivo, em especial aos meus pais Alfonso e Clara por se enorgulharem do meu trabalho.

- Ao meu irmão Carlos e a Hilda que estiveram sempre presentes e me apoiando incondicionalmente.

- Aos meus grandes amigos Gustavo, Paola e Silmara porque estiveram do meu lado em todos os momentos. Vocês fizeram a minha vida no Brasil muito agradável, inesquecível.

- A professora Dra. Neusa Alonso-Falleiros que com muito carinho me brindou sempre seus conselhos e sua mão amiga para me fortalecer e me devolver a confiança.

- A todos aqueles que compartilharam momentos e me ajudaram durante o desenvolvimento desta tese. 


\section{RESUMO}

Quando os materiais ferromagnéticos se submetem a campos magnéticos variáveis, experimentam uma mudança na indução que acontece de forma discreta constituindo os saltos de Barkhausen. Estes saltos são conseqüência da interação entre as paredes de domínio se movimentando e os pontos de ancoragem que atuam como barreiras à sua movimentação. Se durante a aplicação do campo variável, coloca-se uma bobina sensora na superfície da amostra, a mudança no fluxo magnético induz uma seqüência de pulsos de tensão elétrica que conformam o sinal de Ruído Magnético de Barkhausen (RMB). Nos últimos anos tem sido publicados trabalhos que mostram que o RMB depende da microestrutura, do teor de carbono, da dureza, do estado de tensões, da deformação, da condição superficial e da anisotropia, fazendo possível utilizá-lo como um método de ensaio não destrutivo para o monitoramento microestrutural de materiais.

Esta tese mostra a aplicabilidade do RMB para avaliar a variação microestrutural em juntas soldadas de aço estrutural ASTM A36.

A soldagem realizou-se em chapas de $6 \mathrm{~mm}$ de espessura com preparação de chanfro em $\mathrm{V}$, com um e dois passes, e com tratamento posterior de alívio de tensões. Fizeram-se a caracterização de microestruturas e microdurezas numa seção transversal da junta soldada. Numa linha na superficie da chapa transversal ao cordão de solda, mediram-se as tensões residuais por difração de raios-X e nas superfícies das chapas, em várias linhas transversais ao cordão de solda, obtiveram-se sinais de RMB.

Os sinais de RMB se analisaram no domínio do tempo pelo valor máximo, o valor acima de $80 \%$ do máximo, o rms, o rms do envelope, a kurtosis, o skewness e a posição de pico. Analisaram-se no domínio da freqüência o espectro de freqüência na banda completa e em diferentes bandas. Fizeram-se também análises em tempo-escala através da transformada wavelet e wavelet packet, a fim de procurar uma separação do efeito da microestrutura e das tensões residuais. Todos os parâmetros analisados foram grafados em função da distância ao centro do cordão. Obtiveram-se também mapas superficiais com os valores dos parâmetros analisados. Na caracterização microestrutural foi possível identificar tamanhos de zona afetada pelo calor (ZAC) diferentes nas amostras de um e dois passes. Verificou-se também que as microdurezas mudaram através da junta soldada, sendo que a maior dureza se apresentou no limite de fusão e a menor dureza se apresentou no fim da ZAC. Em relação ao RMB constatou-se que no limite de fusão tem-se o menor valor do parâmetro, enquanto que no fim da ZAC tem-se o maior valor do parâmetro. Os mapas superficiais permitiram 
identificar a região correspondente à solda. Todas as análises feitas mostraram a forte influência da mudança microestrutural. Dos resultados obtidos conclui-se que o RMB pode ser usado como método de inspeção não destrutivo para monitorar juntas soldadas reais de aços carbono.

Palavras Chaves: Ruído Magnético de Barkhausen. Ensaios Não Destrutivos Magnéticos. Soldagem de Aços Carbono. Magnetização em Ferromagnéticos. 


\begin{abstract}
Ferromagnetic materials submitted to variable magnetic fields experience discontinuous changes in the induction, being the sudden magnetic flux changes referred as Magnetic Barkhausen Noise (MBN). Fast movements of magnetic domain walls and their interaction with the pinning sites result in the MBN. The corresponding signals are detected as voltage pulses when a pick-up coil is placed in the surface of ferromagnetic materials. In this context, several studies have been recently reported showing the dependence of the MBN on the microstucture, hardness, carbon quantity, residual and applied stress, strain, deformation, surface condition and anisotropy. These features make the MBN an important nondestructive testing technique for material microstructural monitoring.

This work shows results for the evaluation of ASTM A36 carbon steel welded plates by MBN. V-groove shape welded samples were made in a $6 \mathrm{~mm}$ thickness plates, with one-pass and two-pass, and post-weld heat treating for stress relief. Microstructural characterization and microhardness were made on a transversal section of welds. The residual stress were measured by X-ray diffraction. The MBN were measure on surface plates. MBN signals were analyzed on the time domain through several techniques: the maximum value, threshold of $80 \%$ above of maximum, the root mean square ( $\mathrm{rms}$ ), the root mean square of the profile, the kurtosis, the skewness and the profile peak position. The spectra in the entire band and in different width bands were analyzed on the frequency domain. To separate the microstructure effect from that of the residual stress, the wavelet and wavelet packet transform, on the timescale domain, were used. All parameters were plotted in function of the distance to center bead. The microstructural characterization identified different HAZ sizes for one-pass and two-pass. In addition, changes on microhardness throughout welded zones were verified. Greater hardness occurred in the fusion boundary whereas lower microhardness happened in the HAZ end. With MBN monitoring were verified that the MBN lower value happened in the fusion boundary, whereas the MBN bigger value happened in the HAZ end. The surface maps identified the weld region. The study showed that changes in the microstructure influence all analyzed parameters. Consequently, we can conclude that the MBN is a potentially important tool to be used in nondestructive testing for carbon steel weld monitoring.
\end{abstract}

Key Words: Magnetic Barkhausen Noise. Magnetic Non-destructive Testing. Carbon Steel Welding. Ferromagnetic Magnetization. 


\section{LISTA DE SIMBOLOS E ABREVIATURAS}

$a \quad$ Parâmetro de rede

$\mathrm{A}_{3}-\mathrm{A}_{1} \quad$ Temperaturas de austenização $\left({ }^{\circ} \mathrm{C}\right)$

ABM Asociação Brasileira de Metalurgia e Materiais

B Indução magnética (Tesla)

$\mathrm{B}_{\mathrm{r}} \quad$ Remanescência (Tesla)

$\mathrm{B}_{\mathrm{s}} \quad$ Indução magnética de saturação (Tesla)

$\mathrm{cA}_{\mathrm{j}} \quad$ Coeficiente de aproximação da transformada wavelet no nivel $\mathrm{j}$

$\mathrm{cD}_{\mathrm{j}} \quad$ Coeficiente de detalhe da transformada wavelet no nivel $\mathrm{j}$

DEP Densidade Espectral de Potência

$\mathrm{dM} / \mathrm{dt} \quad$ Taxa de magnetização

E Energia no material ferromagnético

$e \quad$ Envelope do sinal

$\mathrm{E}_{\mathrm{an}} \quad$ Energia de anisotropia

$\mathrm{E}_{\mathrm{dis}} \quad$ Energia de desordem

$\mathrm{E}_{\mathrm{ex}} \quad$ Energia de troca

$\mathrm{E}_{\mathrm{m}} \quad$ Energia magnetostática

END Ensaios não destrutivos

F $\quad$ Força de ancoragem

$f \quad$ Freqüência $(\mathrm{Hz})$

$\mathrm{F}_{\text {crit }} \quad$ Força de ancoragem crítica

$\mathrm{F}_{\mathrm{d}} \quad$ Densidade de paredes de domínio

$\mathrm{H} \quad$ Campo magnético $(\mathrm{A} / \mathrm{m})$

Hc Campo coercitivo $(\mathrm{A} / \mathrm{m})$

$\mathrm{H}_{\text {dem }} \quad$ Campo desmagnetizante $(\mathrm{A} / \mathrm{m})$

$\mathrm{H}_{\mathrm{p}} \quad$ Campo magnético de ancoragem $(\mathrm{A} / \mathrm{m})$ 
$k \quad$ Kurtosis

LADIN Laboratório de Dinâmica e Instrumentação

$\mathrm{P}_{\mathrm{a}} \quad$ Probabilidade de aniquilação de domínios

$\mathrm{P}_{\mathrm{g}} \quad$ Probabilidade de crescimento dos domínios magnéticos

$\mathrm{P}_{\mathrm{n}} \quad$ Probabilidade de nucleação de domínios magnéticos

$P(v) \quad$ Distribuição de probabilidade de amplitude do RMB

$P(T) \quad$ Distribuição de probabilidade da duração do sinal RMB

$P(S) \quad$ Distribuição de probabilidade do tamanho do sinal RMB

RMB Ruído Magnético de Barkhausen

rms Root Mean Square

s $\quad$ Representa o sinal de RMB

SMAW Shielded Metal Arc Welding. Processo de soldagem de arco elétrico com eletrodo revestido

$\mathrm{T} \quad$ Temperatura $\left({ }^{\circ} \mathrm{C}\right)$

TIG Tungten Inert Gas. Processo de soldagem com eletrodo de tungstenio e proteção de gas inerte.

$\mathrm{T}_{\mathrm{r}} \quad$ Temperatura arbitraria na qual se avalia o resfriamento

$\mathrm{t}_{\mathrm{r}} \quad$ Tempo de resfriamento

$\mathrm{T}_{\mathrm{s}} \quad$ Temperatura de transformação importante na soldagem

t $\quad$ Tempo (s)

$\mathrm{T}_{\mathrm{m}} \quad$ Temperatura máxima $\left({ }^{\circ} \mathrm{C}\right)$

$t_{s} \quad$ Tempo de permanência acima de uma temperatura $T_{s}$

V Tensão

$\mathrm{V}_{\text {kur }} \quad$ Kurtosis do RMB

$\mathrm{V}_{\max } \quad$ Voltagem máxima de RMB

$\mathrm{V}_{\mathrm{pp}} \quad$ Posição de pico do envelope do RMB

$\mathrm{V}_{\text {rms }} \quad$ rms da voltagem do RMB 
$\mathrm{V}_{\text {rmse }} \quad$ rms do envelope do RMB

$\mathrm{V}_{\text {skew }} \quad$ Skewness do RMB

$\mathrm{V}_{\text {tremax }} \quad$ Média de valores acima de $80 \% \mathrm{~V}_{\max }$ de $\mathrm{RMB}$

$v_{r} \quad$ Velocidade de resfriamento numa determinada temperatura

LF Limite de fusão

ZAC Zona afetada pelo calor

ZF Zona de fusão

ZTP Zona de transformação parcial

$\mathrm{d} \varphi / \mathrm{dt} \quad$ Fluxo magnético na bobina

$\gamma \quad$ Energia superficial das paredes de domínio

$\delta \quad$ Profundidade da camada superficial na qual o campo magnético se atenua, pelo "efeito de pele"

$\delta_{\mathrm{w}} \quad$ Espessura da parede de domínio

$\mu \quad$ Permeabilidade

$\mu_{0} \quad$ Permeabilidade no vazio $\left(4 \pi \times 10^{-7} \mathrm{H} / \mathrm{m}\right)$

$\mu_{\mathrm{r}} \quad$ Permeabilidade relativa

$\sigma \quad$ Condutividade elétrica $\left(\Omega^{-1} \mathrm{~m}^{-1}\right)$

$\sigma_{\mathrm{m}} \quad$ Tensão residual máxima

$\sigma_{\mathrm{x}} \quad$ Distribuição de tensão residual longitudinal

$\sigma_{\mathrm{y}} \quad$ Distribuição de tensão residual transversal

$\Psi(\mathrm{x}) \quad$ Função wavelet mãe 


\section{LISTA DE FIGURAS}

Página

Figura 1 Curva de temperatura em função do tempo para um ponto 26 determinado perto da solda. [1]

Figura 2 Distribuição da temperatura em função da distância ao centro da solda. [1]

Figura 3 Curvas de temperatura-tempo obtida para diferentes pontos desde o centro da solda. [1]

Figura 4 Ciclos térmicos sucessivos numa soldagem multipasse. a) num ponto próximo ao passe de raiz e b) num ponto próximo ao último passe. [1]

Figura 5 Evolução de tensões residuais com a mudança de temperatura numa junta a topo. [3]

Figura 6 Distribuição esquemática de tensões residuais longitudinais e transversais numa junta a topo de um passe. [3]

Figura 7 Curva de magnetização de um aço 3\% Si - Fe. [7]

Figura 8 Curva de histerese típica de um material ferromagnético. [11]

Figura 9 Representação esquemática de: a) magnetização no anel de histerese, b) probabilidade de nucleação -Pn-, aniquilação -Pa- e crescimento -Pg- de domínios, c) densidade de paredes de domínio -Fd-. [12]

Figura 10 Relação dos domínios magnéticos com o processo de magnetização.

Figura 11 Descontinuidades de Barkhausen na curva de magnetização. [13]

Figura 12 Sinal de ruído magnético de Barkhausen.

Figura 13 Variação de valor máximo do RMB em função do teor de carbono, para dois valores diferentes de taxa de campo magnético. [33]

Figura 14 Variação do valor de pico do RMB em função da tensão mecânica aplicada. [42]

Figura 15 Valor de pico máximo de RMB em amostras submetidas a: a) tensões de tração nas regiões elástica e plástica, b) amostras com $2,5 \%$ de deformação.

Figura 16 RMB em amostras nas condições como-soldado e após tratamento de pós-soldagem. a) valores de pico respeito ao centro do cordão de solda, b) relação de RMB com a dureza. [51]

Figura 17 Esquema da preparação da junta soldada.

Figura 18 Esquema da varredura para as medições na superfície da chapa. 
Figura 19 Mesa $x-y$ que garante o deslocamento do sensor a cada milímetro, controlada pelo computador.

Figura 20 Cadeia de medição do sinal Barkhausen.

Figura 21 Sistema de medição de Ruído Magnético de Barkhausen do

Figura 22 LADIN.

Figura 23 Esquema das sondas empregadas e posição da sonda com relação ao cordão de solda. a) sonda 1, b) sonda 2 , c) sonda 3 .

$\begin{array}{lll}\text { Figura } 24 & \text { Esquema da varredura para as medições na superfície da chapa. } & 63\end{array}$

Figura 25 Esquema de decomposição de um sinal através de wavelet. $\quad 70$

Figura 26 Árvore de decomposição wavelet. 71

$\begin{array}{llllllll}\text { Figura } 27 \text { Algumas funções wavelet mãe } \psi \text { empregadas para a } & 72\end{array}$ transformada discreta de wavelet.

Figura 28 Árvore de decomposição do wavelet packet. 73

Figura 29 Microdurezas nas amostras soldada preliminares. a) um passe, b) 75

Figura 30 Mapa superficial do valor rms das amostras preliminares. a) valor máximo do RMB amostra um passe, b) valor rms do RMB amostra um passe, c) valor máximo do RMB amostra dois passes, d) valor rms do RMB amostra dois passes, e) valor máximo do RMB amostra três passes, e) valor rms do RMB amostra três passes.

Figura 31 Macrografia da junta soldada na amostra de um passe. a) como soldada, b) após tratamento de alívio de tensões T1. Obtida na lupa com baixos aumentos. Ataque Nital 2\%.

Figura 32 Macrografia da junta soldada na amostra de dois passes. a) como soldada, b) após o tratamento de alívio de tensões T1. Obtida na lupa com baixos aumentos. Ataque Nital 2\%.

Figura 33 Micrografia da zona de fusão da amostra de um passe como soldada. Microscopia óptica. Nital 2\%.

Figura 34 Micrografia da linha de fusão (LF) e zona de crescimento de grão da amostra de um passe como soldada. Microscopia óptica. Nital 2\%.

Figura 35 Micrografia na região de grão intermediário da amostra de um passe como soldada. Microscopia óptica. Nital 2\%.

Figura 36 Micrografia na região de grão refinado da amostra de um passe como soldada. Microscopia óptica. Nital 2\%.

Figura 37 Micrografia da zona intercrítica da amostra de um passe como soldada. Microscopia óptica. Nital 2\%.

Figura 38 Micrografia do metal base no afetado. Microscopia óptica. Nital $2 \%$. 
Figura 39 Micrografias no passe de raiz da amostra de dois passes. a) zona de fusão, b) zona de transformação parcial. Ataque Nital $2 \%$.

Figura 40 Microestruturas da amostra de dois passes como soldada. a) microestrutura da zona de fusão no segundo passe na amostra de dois passes. b) microestrutura do limite de fusão e da zona de crescimento de grão (ZGG). Microscopia óptica. Nital 2\%.

Figura 41 Microestruturas da amostra de dois passes como soldada. a) microestrutura da zona de grão intermediário, b) Micrografia da zona de grão refinado (ZGF). Microscopia óptica. Nital 2\%.

Figura 42 Microestruturas da amostra de dois passes como soldada. a) Zona de transformação parcial (ZTP), b) metal base não afetado. Microscopia óptica. Nital 2\%.

Figura 43 Perfis de microdurezas na seção transversal da amostra de um passe.

Figura 44 Perfis de microdurezas na seção transversal da amostra de dois passes.

Figura 45 Sinais típicos de Barkhausen nas diferentes regiões da junta soldada. a) zona de fusão, b) linha de fusão, c) zona de grão fino, d) fim da ZAC, e) metal base.

Figura 46 Valor rms do RMB na amostra de um passe como soldado. a) ao longo de quatro linhas na primeira medição, b) ao longo de sete linhas na repetição da medição.

Figura 47 Valor rms do RMB ao longo de uma linha na amostra de um passe como soldado, com as barras indicando o desvio padrão.

Figura 48 Parâmetros de RMB calculados para a amostra de um passe como soldada. a) valor de pico máximo, b) acima $80 \%$ valor do pico máximo, c) rms do sinal, d) rms do envelope, e) kurtosis, f) skewness, g) posição de pico do envelope.

Figura 49 Mapa superficial de RMB da amostra de um passe como soldada. a) valor máximo, b) valor rms.

Figura 50 Parâmetros de RMB calculados para a amostra de um passe com tratamento de alívio de tensão. a) valor de pico máximo, b) acima $80 \%$ valor do pico máximo, c) rms do sinal, d) rms do envelope, e) kurtosis, f) skewness, g) posição de pico do envelope.

Figura 51 Mapa superficial de RMB da amostra de um passe com alívio de tensão. a) valor máximo, b) valor rms.

Figura 52 Parâmetros de RMB calculados para a amostra de dois passes como soldada. a) valor de pico máximo, b) acima $80 \%$ valor do pico máximo, c) rms do sinal, d) rms do envelope, e) skewness, f) kurtosis, g) posição de pico.

Figura 53 Mapa superficial de RMB da amostra de dois passes como soldada. a) valor máximo, b) valor rms. 
Figura 54 Parâmetros de RMB calculados para a amostra de dois passes $102-103$ com tratamento de alívio de tensão. a) valor de pico máximo, b) acima $80 \%$ valor do pico máximo, c) rms do sinal, d) rms do envelope, e) kurtosis, f) skewness, g) posição de pico.

Figura 55 Mapa superficial da amostra de dois passes com alívio de tensão. a) valor máximo, b) valor rms.

Figura 56 Representação do rms do espectro de freqüências na banda completa e nas diferentes bandas. Amostra de um passe como soldada.

Figura 57 rms do espectro de freqüências nas diferentes bandas da amostra de um passe como soldada. a) banda $100 \mathrm{kHz}, \mathrm{b}$ ) rms do espectro do envelope na banda $100 \mathrm{kHz}, \mathrm{c}$ ) banda $1-3 \mathrm{kHz}$, d) banda 1-10 $\mathrm{kHz}$, e) banda $10-30 \mathrm{kHz}, \mathrm{f}$ ) banda $30-60 \mathrm{kHz}, \mathrm{g}$ ) banda $60-85$ $\mathrm{kHz}, \mathrm{h}$ ) banda $85-100 \mathrm{kHz}$.

Figura 58 Mapa superficial dos valores rms do espectro de freqüências. a) banda $10-30 \mathrm{kHz}, \mathrm{b}) \mathrm{rms}$ do espectro do envelope na banda completa.

Figura 59 Representação do rms do espectro de freqüências na banda completa e nas diferentes bandas. Amostra de um passe com alívio de tensão T1.

Figura 60 rms do espectro de freqüências nas diferentes bandas da amostra de um passe com alívio de tensão. a) banda $100 \mathrm{kHz}, \mathrm{b}$ ) rms do espectro do envelope na banda $100 \mathrm{kHz}$, c) $1-3 \mathrm{kHz}$ d) banda 1$10 \mathrm{kHz}$, e) banda $10-30 \mathrm{kHz}, \mathrm{f}$ ) banda $30-60 \mathrm{kHz}, \mathrm{g}$ ) banda $60-85$ $\mathrm{kHz}, \mathrm{h}$ ) banda $85-100 \mathrm{kHz}$.

Figura 61 Mapa superficial da amostra de um passe com alívio. a) rms do espectro na banda $10-30 \mathrm{kHz}, \mathrm{b})$ rms do espectro do envelope na banda completa.

Figura 62 rms do espectro na banda completa. a) um passe como soldada, b) um passe com alívio de tensão.

Figura 63 rms do espectro de freqüências com relação à distância ao centro do cordão da amostra de dois passes como soldada, nas diferentes bandas.

Figura 64 rms do espectro de freqüências nas diferentes bandas da amostra de dois passes como soldada. a) banda $100 \mathrm{kHz}, \mathrm{b}$ ) $\mathrm{rms}$ do espectro do envelope na banda $100 \mathrm{kHz}$, c) banda $1-3 \mathrm{kHz}, \mathrm{d}$ ) banda $1-10 \mathrm{kHz}$, e) banda $10-30 \mathrm{kHz}, \mathrm{f}$ ) banda $30-60 \mathrm{kHz}, \mathrm{g}$ ) banda $60-85 \mathrm{kHz}, \mathrm{h}$ ) banda $85-100 \mathrm{kHz}$.

Figura 65 Mapa superficial do rms do espectro da amostra de dois passes como soldada. a) banda $10-30 \mathrm{kHz}$, b) $\mathrm{rms}$ do espectro do envelope na banda completa.

Figura 66 rms do espectro de freqüências nas distintas bandas de freqüência da amostra de dois passes com alívio de tensão T1.

$105-106$ 
Figura 67

Figura 68

Figura 69

Figura 70

Figura 71

Figura 72

Figura 73

Figura 74

Figura 75

Figura 76

Figura 77

Figura 78

Figura 79 rms do espectro de freqüências nas diferentes bandas da amostra de dois passes com alívio de tensão. a) banda $100 \mathrm{kHz}, \mathrm{b}$ ) rms do espectro do envelope na banda $100 \mathrm{kHz}$, c) banda $1-3 \mathrm{kHz}, \mathrm{d}$ ) banda $1-10 \mathrm{kHz}$, e) banda $10-30 \mathrm{kHz}, \mathrm{f}$ ) banda $30-60 \mathrm{kHz}, \mathrm{g}$ ) banda $60-85 \mathrm{kHz}, \mathrm{h}$ ) banda $85-100 \mathrm{kHz}$.

Mapa superficial de RMB da amostra de dois passes com alívio de tensão. a) rms do espectro na banda $10-30 \mathrm{kHz}$, b) rms do espectro do envelope na banda completa.

rms do espectro na amostra de dois passes. a) como soldada, b) com alívio de tensão.

Coeficientes de aproximação e detalhe reconstruídos da wavelet Daubechies 3, de um sinal de RMB da amostra de um passe como soldada, decomposto em cinco níveis.

Coeficientes de aproximação (à esquerda) e detalhe (à direita) de diferentes wavelet através de uma linha da junta soldada de um passe como soldada. a) wavelet Daubechies, b) wavelet Coiflets, c) wavelet Meyer.

Entropia wavelet na junta soldada de uma linha da amostra de um passe como soldada. a) entropia Shannon, b) entropia log energy c) entropia trhesholding.

Árvore de decomposição do sinal de RMB através da wavelet packet. a) árvore de decomposição completa, b) árvore que melhor representa o sinal.

Valores rms dos coeficientes de aproximação e detalhe correspondente a árvore de decomposição wavelet packet da amostra de um passe como soldada.

Tensão residual medida por difração de raios-X. a) amostras de um passe, b) amostras de dois passes.

Comparação dos valores Vrms das amostras como soldada e com tratamento de alívio de tensão T1. a) amostra de um passe, b) amostra de dois passes.

Comparação dos valores Vrms das amostras como soldada e com tratamento de alívio de tensão T2. a) amostra de um passe, b) amostra de dois passes.

Valor rms do RMB na linha de difração de raios-X na amostra de um passe como soldada e com tratamento de alívio de tensão T1 e T2, medidas com a sonda 3 .

Valor rms do RMB na linha de difração de raios-X na amostra de dois passes como soldada e com tratamento de alívio de tensão T1 e T2, medidas com a sonda 3 .
$115-116$ 


\section{LISTA DE TABELAS}

Página

Tabela 1 Valores de "efeito de pele" em materiais ferrosos, assumindo $\mu_{\mathrm{r}} \approx 50-5000$ e $\sigma=\left((5-10) \times 10^{6} \Omega^{-1} \mathrm{~m}^{-1}\right.$. [11]

Tabela 2 Profundidade aproximada do sinal de RMB, para diferentes freqüências de análise, em aço EN36 endurecido superficialmente.[20]

Tabela 3 Parâmetro de soldagem empregados nas amostras preliminares.

Tabela 4 Características das sondas utilizadas.

Tabela 5 Composição química do aço estrutural ASTM A36.

Tabela 6 Características do electrodo E7018.

60

60

Tabela 7 Parâmetros de soldagem das amostras definitivas

61

Tabela $8 \quad$ Tratamentos térmicos pós-soldagem feitos nas amostras. 61

Tabela 9 Parâmetros empregados para a medição de difração de raios-X. $\quad 63$

$\begin{array}{lll}\text { Tabela } 10 & \text { Tamanhos da zona de fusão e zona afetada pelo calor nas } & 74\end{array}$ amostras soldadas preliminares.

Tabela 11 Valores de microdureza das amostras preliminares de um, dois e três passes.

Tabela 12 Microdurezas na seção transversal da amostra de um passe. Carga $100 \mathrm{~g}$.

Tabela 13 Microdurezas na seção transversal da amostra de dois passes. Carga $100 \mathrm{~g}$.

Tabela 14 Profundidades de emissão dos sinais de RMB segundo a banda de freqüência analisada.

Tabela 15 Bandas de freqüência de aproximação e detalhe em cada nível da decomposição.

Tabela 16 Bandas de freqüência dos coeficientes de aproximação e detalhe da decomposição do sinal de RMB por wavelet packet. 